Relations industrielles

Industrial Relations

\title{
La responsabilité civile des centrales syndicales - Jugements de la Cour Suprême du Canada dans la cause Gaspé Copper Mines vs United Steel Workers of America
}

Volume 25, numéro 1, 1970

URI : https://id.erudit.org/iderudit/028107ar

DOI : https://doi.org/10.7202/028107ar

Aller au sommaire du numéro

Éditeur(s)

Département des relations industrielles de l'Université Laval

\section{ISSN}

0034-379X (imprimé)

1703-8138 (numérique)

Découvrir la revue

Citer cet article

(1970). La responsabilité civile des centrales syndicales - Jugements de la Cour Suprême du Canada dans la cause Gaspé Copper Mines vs United Steel Workers of America. Relations industrielles / Industrial Relations, 25(1), 132-151.

https://doi.org/10.7202/028107ar

Tous droits réservés (C) Département des relations industrielles de l'Université Laval, 1970
Ce document est protégé par la loi sur le droit d'auteur. L'utilisation des services d'Érudit (y compris la reproduction) est assujettie à sa politique d'utilisation que vous pouvez consulter en ligne.

https://apropos.erudit.org/fr/usagers/politique-dutilisation/ 


\title{
La responsabilité civile des centrales syndicales
}

\author{
JUGEMENT DE LA COUR SUPRÊME DU CANADA DANS LA \\ CAUSE GASPÉ COPPER MINES VS UNITED STEEL WORKERS \\ OF AMERICA *.
}

\section{LE JUGE FAUTEUX}

L'appelante, United Steel Workers of America, a été condamnée le 7 décembre 1964, par M. le juge Lacoursière de la Cour supérieure de la province de Québec, à payer à l'intimée, Gaspé Copper Mines Limited, \$1,747,695 avec intérêts à compter du 31 décembre 1957. Ce montant fut accordé à l'intimée en réparation du préjudice lui résultant d'une grève illégale, déclarée le 10 mars 1957 et prolongée jusqu'au début d'octobre de la même année, et de la commission, au cours de cette grève, de divers délits et offenses criminelles.

United Steel Workers of America appela de ce jugement, et ce, tant sur la question de responsabilité que sur celle du quantum des dommages. De son côté, Gaspé Copper Mines Limited logea un contre-appel quant au quantum. Avant l'audition de l'appel, en Cour du Banc de la Reine, les parties s'entendirent sur le montant des dommages subis par l'intimée, en raison des faits ci-dessus et consignèrent cet accord dans une admission libellée comme suit : (Traduction)

Les parties admettent :-

Qu'il faut corriger le calcul des dommages au jugement de la Cour supérieure, rendu le 7 décembre 1964, de façon à réduire ces dommages à la somme de $\$ 1,646,057.00$, plus intérêts sur ladite somme

* Jugement prononcé le 27 janvier 1970 coram Juges Fauteux, Abbot, Martland, Judson et Hall. Motifs du jugement par le Juge Fauteux; souscrivant à son avis, Juges Abbot, Martland et Judson. Dissidence du Juge Hall.

** On trouvera dans Relations industrielles d'autres renseignements concernant cette affaire.

1. «La responsabilité civile des centrales syndicales : extraits du jugement du juge Lacoursière dans la cause Gaspé Copper Mines vs United Steel Workers of America 》, Relations industrielles, vol. 22, no 1, 1965, pp. 150-187.

2. «Pouvoir de la C.R.O. en matière de reconnaissance syndicale 》 dans Relations industrielles, vol. 12, no 3, 1957, pp. 253-257.

3. "Juridiction de la C.R.O. », Relations industrielles, vol. 12, no 4, 1957, pp. 385-392.

4. Chartier, Roger, «Murdochville : les faits》 Relations industrielles, vol. 12, no 4, 1957, pp. 374-381.

5. Gosselin, Emile, «La marche sur Murdochville» Relations industrielles, vol. 12, no 4, 1957, pp. 382-383.

6. «Murdochville : dernière pièce au dossier »Relations industrielles, vol. 13, no 2, 1958, p. 232. 
au taux de 5 p. 100 l'an à compter du 31 décembre 1957, conformément au tableau et aux notes annexés; que le montant ainsi modifié constitue un chiffre exact des dommages subis par la demanderesse par suite des faits mentionnés audit jugement, de sorte que l'estimation des dommages n'est plus en litige dans la présente cause, la responsabilité totale ou partielle quant à ces dommages restant le seul point à déterminer.

Cette admission ne comporte aucun aveu de responsabilité de la part de la défenderesse-appelante quant à ces dommages. Elle est faite sous la condition expresse que, sur la question de la responsabilité, les parties se réservent le droit d'invoquer toute la preuve, y compris celle relative au montant des dommages, pour établir leurs prétentions respectives.

La présente admission liera les parties, tant dans le présent appel que dans tout appel ultérieur.

L'appel, ne portant alors que sur la question de responsabilité, fut entendu par MM. les juges Hyde, Taschereau et Brossard. Après une audition qui dura dix-neuf jours, - le procès, où environ 350 témoins furent entendus et 800 exhibits produits, ayant lui-même duré 120 jours, - l'affaire fut prise en délibéré et ultérieurement décidée par un jugement rendu le 16 mars 1967. Sur le quantum, on donna effet à l'admission des parties et réduisît, en conséquence, le montant des dommages à la somme de $\$ 1,646,057.00$. Sur la responsabilité, on fut unanime à confirmer, en substance, les conclusions retenues par le juge au procès, savoir que la déclaration de cette grève le 10 mars 1957 et sa continuation jusqu'aui début d'octobre de la même année, étaient nettement illégales et délictuelles; que cette grève avait été fomentée, organisée, dirigée, soutenue et financée par des agents et représentants de l'appelante; que les actes de piquetage illégal, le blocus du 10 au 19 mars à l'entrée de l'usine, les actes de violence, de dynamitage, de sabotage, d'intimidation, d'obstruction et violations des termes d'une injonction de la Cour supérieure, étaient autant d'actes délictuels ou criminels qui, collectivement et indépendamment de l'entrée en grève et de son maintien, avaient été l'une des causes de l'interruption totale ou partielle de la production de l'entreprise de la compagnie intimée et autant d'actes commis avec la participation, l'approbation expresse ou tacite, les encouragements, les incitations ou les appuis matériels et financiers des agents et représentants de la haute hiérarchie et direction de l'appelante. En droit, on a conclu, comme en Cour supérieure, que l'appelante devait répondre de tous les dommages, tant ceux résultant du fait particulier de la grève que ceux résultant de ces actes délictuels ou criminels. Toutefois, en ce qui concerne les dommages attribuables spécifiquement au fait particulier de la grève, $M$. le juge Brossard, pour sa part, considéra que la compagnie avait une part de responsabilité dans le fait de son déclenchement le 10 mars 1957 et devait, pour cette raison, supporter, dans une proportion qu'il fixa à $25 \%$, le préjudice lui résultant de ce chef.

De là le pourvoi de United Steel Workers of America contre ce jugement de la Cour du Banc de la Reine.

L'action instituée par l'intimée à l'appelante se fonde sur les articles 1053 et $1054 \mathrm{du}$ Code civil de la province de Québec. Il est classique, élémentaire de dire 
que pour soutenir une telle action, le fait dont on se plaint doit être illicite, dommageable au demandeur et imputable au défendeur. En l'espèce, personne évidemment ne saurait pour un instant mettre en doute le caractère délictuel et dommageable de cette grève, faite en violation des dispositions de l'article 24 de la $\mathbf{L o i}$ sur les Relations Ouvrières, (S.R.Q. 1941, c. 162A), aussi bien que des actes commis au cours de cette grève. Sur le point, il y a accord des deux cours inférieures et on reconnaît à l'admission plus haut citée, que la totalité des dommages subis furent causés par l'ensemble des faits mentionnés au jugement, ce qui inclut et le fait de la grève et les actes délictuels ou criminels. Aussi bien, la seule question en litige, en cet appel, porte-t-elle sur l'imputabilité. Encore faut-il préciser que la question, à mon avis, se limite à savoir si, comme en ont jugé la Cour supérieure et la Cour d'appel, l'appelante doit seule supporter tous les dommages subis par l'intimée ou si, suivant l'opinion de M. le juge Brossard, l'intimée doit être tenue conjointement responsable du déclenchement de la grève et supporter une part du préjudice lui en résultant. En somme, il s'agit d'abord de déterminer si, en fait, la compagnie intimée a provoqué l'entrée en grève par ses agissements, soit en instituant et participant à la suspension des procédures de prohibition ou soit en procédant à ce qu'on a appelé la mise à pied du président du syndicat local de l'appelante et, dans l'affirmative, déterminer si, par suite et en droit, la compagnie intimée doit être tenue conjointement responsable de l'entrée en grève et supporter une partie du préjudice attribuable à la grève elle-même.

Voyons sommairement les faits et circonstances essentiels dans le contexte desquels se pose la question ainsi définie.

Organisée à cette fin en 1950, la compagnie intimée exploite une entreprise minière à Murdochville, dans la péninsule de Gaspé, province de Québec. Filiale de la compagnie Noranda Mines Limited qui est alors propriétaire de $95 \%$ de son capital-actions, elle a son bureau chef à Noranda, province de Québec, et reçoit ses directives du bureau de la compagnie Noranda à Toronto. Vers la fin de l'année 1955, la compagnie intimée compte environ 1,000 employés avec lesquels elle est liée par une convention collective non automatiquement renouvelable. Cette convention, intervenue en 1954 entre elle et le Local 544 de l'Union Internationala des Employés de Mines du District de Gaspé, doit expirer le 15 juillet 1956. Au printemps de 1956, ce syndicat local, affilié au Congrès des Métiers du Travail, ne compte plus qu'une vingtaine de membres. À cette époque, en avril, une entento intervient à Toronto entre les officiers du Congrès et ceux de l'appelante. On envisage la dissolution du Local 544 affilié au Congrès et convient que l'appelante aura désormais le champ libre pour établir son propre local à Murdochville. Comme l'appelante représente déjà, par son Local 4278, les travailleurs de Noranda, dont le contrat de travail expire lui-même en octobre, - on pourra ainsi, par l'action conjointe des deux unités syndicales, assurer une position de commande dans la négociation imminente des conventions collectives.

À la suite de l'entente ci-dessus, l'appelante, sans tarder, délègue à Murdochville trois représentants dont son organisateur Roger Bédard, pour aider à l'établissement et à l'organisation de son nouveau local auquel elle décernera le 21 mai 1956 une charte d'affiliation. Le Local 544 du Congrès des Métiers du Travail est dissout et Théo Gagné qui en était le président devient président du nouveau syndicat désigné comme Local 4881 de l'appelante. Le premier juin suivant, le syndicat transmet 
à la Commission des Relations Ouvrières une requête pour être accrédité comme représentant des travailleurs de la compagnie intimée. La Commission en avise celle-ci le 22 juin. Le 3 juillet, elle ordonne une enquête sur le caractère représentatif du Local, enquête qui, à une date imprécise en juillet, aurait, suivant le rapport fait à la Commission par l'un de ses inspecteurs, établi que sur 920 salariés de la compagnie, éligibles comme membres du Local, 739 étaient des membres 《déclarés» à l'Union. Le 6 juillet, les avocats de la compagnie écrivent à la Commission pour obtenir copie de la requête du syndicat, de la résolution et autres documents produits à l'appui. Le 15 juillet, la Commission fait droit à leur demande mais refuse, «suivant la pratique suivie par la Commission». dit-elle, de fournir les noms apparaissant sur ces documents. Au motif qu'elle a droit à cette information et que le refus de la lui donner équivaut à la négation d'une juste audition. La compagnie obtient le 2 août 1956, sur la foi d'une requête appuyée d'un affidavit et présentée ex parte le jour précédent, la délivrance d'un bref introductif d'instance de prohibition, enjoignant à la Commission et au syndicat local de surseoir à toutes procédures relatives à cette demande de reconnaissance syndicale et de comparaître, le vingtième jour après la signification du bref, pour répondre à la demande contenue dans la requête. La Commission et le syndicat comparaissent le 22 et le 23 août respectivement. Le 10 septembre 1956, la Commission oppose une inscription en droit totale au bref de prohibition et ce n'est qu'un an après, soit le 27 septembre 1957, que M. le juge Morin, à qui elle fut soumise, en dispose. Il accueille cette inscription en droit et rejette le bref de prohibition. À la fin de ses notes, le savant juge explique ce délai d'un an :

Avant que de conclure le présent jugement, le tribunal tient à faire une mise au point.

Dans cette cause, l'inspection en droit fut soumise au Tribunal à la fin de septembre, 1956. Le procureur de la requérante et le procureur de l'intimée demandèrent au Tribunal l'autorisation de plaider par écrit sur l'inscription en droit à l'encontre de la requête de la requérante. Le 2 novembre, 1956, Me Victor Trépanier, le procureur de la Commission de Relations Ouvrières de la Province de Québec, l'intimée, produisait son plaidoyer par écrit. Après la production de ce plaidoyer, il fut convenu entre le procureur de la Commission de Relations Ouvrières de la Province de Québec, l'intimée, et Me Gaston Pouliot, le procureur de Gaspé Copper Mines, Limited, la requérante, d'attendre le jugement de la Cour d'Appel dans deux causes où la question discutée dans la présente cause avait été soumise à un banc de sept juges de la Cour d'Appel. La Cour d'Appel rendit jugement dans l'une de ces causes dans le cours du mois d'août, 1957, sans toutefois se prononcer sur la question dont il s'agit dans la présente cause. Quant à la deuxième cause également entendue par un banc de sept juges, la Cour d'Appel n'a pas encore rendu sa décision.

À cause de certaines critiques, le Tribunal décida de ne pas attendre la décision de la Cour d'Appel dans la deuxième cause à elle soumise. Il exigea du procureur de la requérante, Gaspé Copper Mines, Limited, son plaidoyer par écrit. Ce plaidoyer écrit fut envoyé au Tribunal le 10 septembre 1957.

Le Tribunal croit devoir faire cette mise au point à la suite de certaines critiques injustes pour démontrer qu'il n'y a eu dans toute 
cette affaire aucun retard intentionnel non seulement de la part du Tribunal, mais aussi de la part des procureurs des parties dans la présente cause.

Quant au procureur de la mise-en-cause (le syndicat), Me G. Merril Désaulniers, il produisit une comparution dans l'action. Il n'a pas jugé à propos cependant de produire un plaidoyer écrit.

En Cour d'appel, on a noté que le syndicat ne fut pas partie à l'accord pour suspendre la procédure mais que, au regard du dossier, rien n'indique qu'on ait fait de sa part des démarches judiciaires pour se plaindre de cette suspension, demander l'adjudication de l'inscription en droit ou contester l'émission du bref de prohibition. Et pourtant, tant qu'il n'était pas disposé de l'ordonnance de prohibition, la Commission ne pouvait procéder sur la requête en accréditation et les dispositions de l'article 24 de la Loi sur les Relations Ouvrières défendaient formellement aux travailleurs de la compagnie intimée de recourir à la grève avant l'accréditation et avant que ne soient épuisées les procédures de conciliation, d'arbitrage et un délai de 14 jours après la sentence arbitrale. Pour contraindre la compagnie à reconnaître le syndicat comme représentant accrédité, on opta éventuellement pour d'autres moyens, soit la grève, suivie des délits et actes criminels que l'on sait. Avant de référer aux circonstances afférentes au déclenchement de cette grève le 10 mars 1957, disons pour compléter ce récit sur la procédure, bien que ceci n'ait de portée sur la question à déterminer, qu'à l'expiration du délai prescrit pour appeler du jugement de M. le juge Morin, soit le 24 octobre 1957, la compagnie produisit une contestation de la demande d'accréditation et le 6 février 1958, la Commission rejeta cette demande pour des motifs ainsi résumés aux notes de $M$. le juge Brossard, savoir :
... que depuis la demande, nonobstant la suspension des procédures de la Commission par ordonnance de cour, le Local ayant procédé à faire une grève illégale au cours de laquelle nombre d'actes illégaux ont été commis, le Local n'a plus le caractère de bonne foi requis par la loi pour son accréditation et qu'il ne groupe pas la majorité des salariés de la compagnie.

Faisant obstacle à la reconnaissance du droit qu'on prétendait avoir à l'accréditation et au plan qu'on s'était tracé en vue de la négociation des conventions collectives à Murdochville et à Noranda, ces procédures de prohibition étaient, sans doute, frustrantes. Le conflit, qui allait éventuellement conduire à la grève, ne tarda pas à s'amorcer, s'il ne l'était déjà, pour s'aggraver avec le temps, favorisé et activé qu'il était par les déclarations, incitations faites aux membres de ce syndicat, peu versés dans la conduite des activités syndicales, par les agents et représentants expérimentés de l'appelante. Déjà avant l'institution de ces procédures, Bédard avait, à une assemblée des membres du Local, souligné le fait que 650,000 employés des aciéries américaines étaient en grève bien que leurs conditions de vie étaient vingt ans en avance sur celles prévalant au Québec et que la grève demeurerait le moyen pour continuer à marcher avec le progrès. À l'assemblée du 30 août, il leur lisait quelques «passages cocasses » du bref de prohibition et leur disait que «devant une telle effronterie de la part de la compagnie, il ne leur restait que l'arme efficace qu'ils possèdaient: la grève ». À la même assemblée, Théo Gagné, de son côté, insiste sur la nécessité de prendre un vote de grève et on 
décide qu'on y procédera dans une dizaine de jours, ce qui, en fait, se produit à l'assemblée spéciale du 20 septembre. Avant de prendre le vote, Bédard passe en revue les événements des trois dernières semaines, explique l'urgence qu'il y a pour eux de prendre immédiatement ce vote, annonce qu'un million et demi de dollars est prévu par C.I.O. et C.L.C. dans l'éventualité de conflits à Murdochville et à Noranda et réfère aux négociations en cours à Noranda et à la promesse du Local de Noranda de ne pas signer de contrat de travail tant que le Local de Murdochville n'aura pas signé le sien. Le vote est pris; le compte est de 667 votes en faveur de la grève, 12 contre et 2 nuls. Prévoyant le déclenchement éventuel d'une grève, la compagnie procède à faire provision de vivres et aménagement de lits, literie, etc., en vue d'un blocus, d'un siège susceptible d'être subi par les non-grévistes à l'intérieur de ses usines, siège qui ne manquera pas d'être établi et maintenu du 10 au 19 mars pour être suivi, du 19 mars au 25 avril, d'un piquetage illégal. Le 27 septembre, les officiers du Local adressent une lettre au gérant de la compagnie pour l'informer qu'à l'assemblée du 20 septembre on a décidé de requérir la compagnie de rencontrer le comité de négociation du Local et qu'à ces fins ce comité sera disponible sur avis de trois jours; on ajoute que $95 \%$ des employés de la compagnie sont membres en règle du Local. Le 1er octobre la compagnie répond qu'après avoir sérieusement considéré cette demande du Local, elle en est venue à la conclusion qu'elle ne pouvait rencontrer le comité pour négocier, vu l'action qui était pendante en Cour supérieure relativement à l'accréditation. Le même jour, la compagnie annonce officiellement une augmentation générale de salaire, accompagnée d'augmentations particulières déjà projetées et sous étude depuis le début de septembre. Effectuées sans négociations, ces augmentations, mises en vigueur le même jour, sont acceptées par tous les travailleurs.

Au début de février 1957, la Direction de la compagnie, afin d'améliorer le rendement de la production, prend des mesures pour hâter la fin de certains travaux de construction poursuivis sous terre. On estime, à la Direction, qu'à la fin de ces travaux, et après l'installation de certaines machineries lourdes, le nombre des employés pourra être substantiellement réduit. Gagné est lui-même, à titre de plombier de première classe, affecté à ces travaux depuis près de deux ans. À la même époque, il s'absente, malgré la défense de son contremaître, qui le juge indispensable à la poursuite efficace de ces travaux, pour aller à Québec assister à une convention de diverses unions ouvrières dont celle de l'appelante. Au cours de cette visite, il rencontre certaines autorités de l'appelante qui offrent de mettre à la diposition du Local de Murdochville une ou plusieurs tentes et un certain nombre de chaudrons pour être utilisés par les piqueteurs, advenant l'entrée en grève. De retour à Murdochville, Gagné cherche à justifier son absence en remettant au directeur général de l'usine, William G. Brissenden, un certificat médical inexact. Conscient du subterfuge, celui-ci s'abstient de recourir à des mesures disciplinaires et ferme les yeux pour éviter la possibilité de troubles avec l'Union.

À une assemblée tenue le 19 février, Pat Burke, un représentant régional de l'appelante, fait, suivant le procès-verbal de cette assemblée, la déclaration suivante aux membres du Local :

Nous devons être prêts (sic) à former nos lignes de piquettage (sic) en aucun temps après le 10 mars. La compagnie n'améliora (sic) pas vos conditions tant qu'elle ne s'y verra pas forcée par la grève. 
Il se peut qu'en cette citation, il faille lire nos conditions plutôt que vos conditions. Vers le même temps, Gagné fait certaines déclarations qu'un des travailleurs, Pierre Lecrin, entendu comme témoin, rapporte comme suit :

Q. Voulez-vous dire à la Cour ce que Théo Gagné vous a déclaré dans le mois de janvier ou février mil neuf cent cinquante-sept (1957) ? R. Bien, il m'a déclaré...

Q. Parlez à la Cour. R. Comme quoi il était revenu de Québec et puis là à son retour, il a dit que tout était décidé pour la grève. Il dit «ce sera fait entre le dix (10) de mars ou le dix (10) d'avril, il dit, dans ce temps-là, la date, y'a pas de date de fixée, mais ce sera dans le courant de ce mois-là, si la compagnie ne nous rencontre pas avant ça, et je ne pense pas, il dit, depuis le temps que ça dure, je ne pense qu'elle va nous rencontrer. Donc, préparez-vous, les gars, ça va se faire dans ce temps-là ».

Q. Et Gagné revenait de Québec ? R. C'est ce qu'il m'a dit.

Q. Et est-ce qu'il vous a dit qui il avait vu à Québec? Qui il avait rencontré ? R. Les autorités de l'Union. Il n'a pas nommé personne.

On a noté, en Cour d'appel, qu'au début de février, la possibilité du déclenchement d'une grève légale à Noranda pouvait être envisagée pour à peu près le 10 mars, compte tenu des délais prévus à l'article 24 de la Loi sur les Relations Ouvrières. En fait, cependant, la sentence arbitrale à Noranda ne fut rendue que le 21 mars; on accepta les offres de la compagnie Noranda Mines Ltd. et il n'y eut pas de grève.

Trois jours après que Pat Burke eut fait les déclarations ci-dessus à l'assemblée du 19 février, Brissenden fait venir Gagné à son bureau et cherche, mais en vain, à obtenir de celui-ci la promesse d'un avis préalable à toute décision de recourir à la grève.

Mercredi le 6 ou jeudi le 7 mars, la ou les tentes et chaudrons qu'on avait offerts à Gagné lors de sa visite à Québec, sont expédiés de Val d'Or à Noranda et de là réexpédiés, à l'adresse de Bédard, à Murdochville, où ils arriveront dans la soirée du 9 au 10 mars, pour être utilisés dès lundi, 11 mars.

Vendredi, le 8 mars, dans la matinée, Dempsey, contremaître de Gagné, agissant sur les instructions de Leslie, le directeur de l'atelier d'usinage, remet à Gagné un document intitulé Termination Notice. On remet généralement ce document, désigné sous le nom de Blue Slip, aux ouvriers qui sont congédiés, à ceux qui quittent l'emploi de leur propre accord et, - souvent mais non toujours, - à ceux qui sont assignés à un autre poste ou division de l'usine. Gagné proteste; il invoque sa séniorité. Dempsey lui explique qu'on est arrivé à la fin des travaux de construction, qu'on procède à mettre à pied ceux dont les services ne sont plus requis et que tel est le cas d'un homme de son métier et de sa catégorie. Et, conformément aux instructions qu'il avait reçues de Brissenden, Dempsey dit à Gagné de voir la Direction. Disons incidemment qu'en outre de Gagné, cinq ou six autres ouvriers, charpentiers ou menuisiers, sont mis à pied le même jour. Gagné demeure au travail jusqu'au moment de relève, soit à 3 heures p.m., et va alors remettre à l'un des contremaîtres des mécaniciens les outils qui 
appartiennent à la compagnie. À l'un des employés et officiers du syndicat, Abraham Arsenault, qu'il connaît bien et qu'il rencontre à l'atelier d'usinage, il déclare, suivant le témoignage de ce dernier :

... que là où il travaillait sur le posage de tuyaux la construction était finie et qu'il avait été avisé de se rapporter au bureau pour tâcher d'y trouver un autre emploi.

En fait, anticipant que les services de Gagné ne seraient plus requis aux travaux de construction fait sous terre, et anxieux de le voir assigner à un autre poste dans l'usine, Brissenden avait, plus tôt au cours de la semaine, discuté de la question avec Richard Coleman et John Hall, directeurs d'autres divisions de l'usine, et avait donné instruction à Leslie - qui s'y est conformé - d'attirer sur la question l'attention de George McKerrow, un autre directeur de division, le tout, ainsi que tous en ont témoigné, afin de voir si on ne pouvait trouver une ouverture pour Gagné dans leurs divisions. Prévoyant aussi la possibilité que celui-ci, après avoir reçu le Blue Slip, s'adresse au directeur du personnel, Roger Cyr, Brissenden avait, dans le cours de la journée, téléphoné à ce dernier pour lui dire que, sous aucune circonstance, devait-il, dans le cas de Gagné, procéder à faire ce qu'il est normalement requis de faire dans le cas d'une mise à pied définitive mais qu'il devait plutôt, s'il le voyait, lui dire que Brissenden voulait le voir. Après avoir quitté l'usine, Gagné se rend éventuellement au bureau du syndicat; il y rencontre des confrères et de là, en fin d'après-midi ou début de soirée, il entre en communication, par téléphone, avec Bédard qui se trouve à Newcastle, NouveauBrunswick, et l'informe de ce qui vient de se produire. Suivent, par après, diverses communications téléphoniques entre différents représentants ou agents de l'appelante, soit Bédard à Newcastle, Pat Burke à Noranda, un autre à Montréal et certains inconnus à Toronto où se trouve, pour le Canada, le bureau chef de l'appelante. On fait dire à un des agents de l'appelante, Raymond Lapointe de Montréal, de se rendre immédiatement à Murdochville pour assister Bédard. Raymond Lapointe n'y arrivera, cependant, que le lundi, 11 mars, pour, par la suite, participer à la direction de la grève.

Le lendemain matin, samedi 9 mars, Gagné, comme il y avait été invité la veille par Dempsey, se rend à l'usine pour voir la Direction, en l'occurence Leslie, directeur de la division d'usinage. Chemin faisant, il croise Brissenden et lui demande une entrevue que celui-ci, pressé et retenu par la visite d'un ingénieur étranger, ne peut lui accorder sur le champ et fixe à lundi, 11 mars, dans la matinée ou l'après-midi. Gagné poursuit son chemin, voit Leslie qui lui répète, en substance, ce que Dempsey lui avait dit la veille et lui dit que Brissenden veut le voir; rendez-vous dont Gagné et Brissenden viennent tout juste de convenir pour le lundi. Gagné retourne au bureau du syndicat. Dans l'après-midi, il revient à l'usine. Cette fois, ce n'est pas pour y discuter de son emploi mais pour en retirer ses vêtements de travail et ses propres outils. N'étant pas muni du laissez-passer, depuis toujours exigé de tout travailleur qui se présente à la barrière à des heures autres qu'à ses heures de travail, - comme c'est alors le cas de Gagné qui ne travaille pas les samedis, - on lui refuse l'entrée à l'usine et il doit, pour y accéder, avoir recours au Directeur du personnel qui se rend sur place, lui fait ouvrir la barrière et l'accompagne dans l'usine. 
Alors qu'il se rendait à l'usine, ce samedi après-midi-là pour y chercher ses effets personnels de travail, Gagné savait fort bien que sa mise à pied n'avait rien de définitif et que l'entretien qu'il devait avoir avec Brissenden le lundi avait précisément pour objet de discuter d'un autre poste à l'usine. Cela ressort même du témoignage, par ailleurs inexact sur d'autres points, que Gagné lui-même a donné pour expliquer pourquoi il ne s'était pas rendu au rendez-vous le lundi :

Parce que la façon dont ça s'est passé, j'ai d'abord, je vais donner les raisons pourquoi je ne suis pas allé voir monsieur Brissenden. Y'a le fait que j'ai été arrêté à la barrière par la police, maintenant, le fait au comité, les gens qui se sont réunis m'ont dit et m'ont fait comprendre, et moi-même, je le comprenais que vraiment je n'avais pas de raison d'avoir un traitement de faveur parce que justement il était bien clair que j'étais congédié, monsieur Dempsey m'aurait dit, si j'étais transféré et enfin tout a été énuméré, y'a eu huit (8) charpentiers de congédiés à cette occasion-là, et les huit (8) charpentiers n'avaient pas de rendez-vous avec monsieur Brissenden ou quelqu'un d'autre pour reprendre leur emploi, ils étaient mis à pied à toute fin et monsieur Lebel aussi dans le comité, surtout le comité du dimanche, où je leur ai dit que je devais voir monsieur Brissenden le lundi matin, ils m'ont dit, ils m'ont dit «Théo oublie ça; ça, c'est simplement des histoires, il va peut-être t'offrir un emploi de contremaître ou quelque chose » comme ça m'avait déjà été offert, un emploi de contremaître ; monsieur Dempsey m'avait déjà offert un emploi de contremaître dans l'automne cinquante-six ("56) et j'ai cru qu'il m'offrait cet ennploi-là plutôt parce que j'étais président de l'Union, et enfin, j'ai pas dit non, mais j'ai pas dit oui non plus.

Postérieur à la décision qu'il avait prise d'aller à l'usine pour y chercher ses effets personnels de travail, cet incident de l'arrêt à la barrière, attribuable au fait que Gagné n'avait pas le permis requis pour accéder à l'usine le samedi, ne saurait, de toute évidence, être validement invoqué comme raison de cette décision. - Le juge au procès n'a ajouté aucune foi au témoignage de Gagné, pas plus, soit dit en passant, qu'à celui des principaux représentants ou agents de l'appelante. - Alors pourquoi cette décision de Gagné ce samedi-là ? Pourquoi va-t-il à l'usine dans l'après-midi de samedi chercher ses outils et ses vêtements de travail alors que dans la matinée du même jour, il y était allé pour avoir des explications de sa mise à pied et s'assurer la continuation de son emploi ? Serait-ce que, entre ces deux visites, on l'aurait mis au courant du fait que l'appelante avait pris ou allait imminemment prendre la décision de saisir l'occasion de sa mise à pied pour en faire un prétexte de grève et en autoriser le déclenchement pour le lendemain, dimanche, à minuit, et qu'alors et en raison de ce fait, il n'y avait plus lieu pour lui de poursuivre l'objet du rendez-vous qu'il devait avoir - et qu'évidemment il n'a pas tenu - le lundi matin avec Brissenden et qu'il n'y avait plus lieu, en conséquence, de laisser ses effets personnels à l'usine pour y reprendre incessamment le travail ? Ce même après-midi, samedi 9 mars, on procède à répandre, avec la nouvelle de la mise à pied de Gagné qui depuis la veille avait cours, une rumeur voulant que 100 ou 125 autres employés soient mis à pied. Il se peut que cette rumeur tire son origine dans le fait qu'au début de février, ainsi que déjà mentionné, la Direction envisageait comme possible une réduction substantielle de personnel après la terminaison des travaux de construction faits sous terre et après l'installa- 
tion de la machinerie lourde. Toutefois, on déforme les faits en ce qui concerne la raison et la date de la réduction prévue. On représente, ce qui est faux, que les congédiements doivent se produire dès le début ou au cours de la semaine suivante, que 100 ou 125 employés seront alors congédiés et que parmi eux se trouvent surtout des officiers et stewards de l'Union. La fausseté de cette rumeur a été reconnue en Cour supérieure et en Cour d'appel. Gagné participe activement à la diffusion de cette fausseté, s'il n'en a pas été lui-même le principal ou l'un des principaux artisans. Et le même après-midi, on convoque une assemblée des officiers du syndicat pour le lendemain, dimanche après-midi, et une assemblée générale des membres pour le dimanche soir; on donne avis de ces convocations dans les théâtres le même soir et aux messes le dimanche.

Dimanche, 10 mars, dans la matinée, Gagné se rend au bureau du syndicat et $\mathrm{y}$ fait venir Abraham Arsenault qui relate ainsi ce qui se passe à cette rencontre :

Le dix (10) mars, je suis allé à la messe de bonne heure et puis dans le courant de l'avant-midi, Théo Gagné m'a téléphoné du bureau si je voulais bien monter au bureau qu'il avait une affaire importante à me parler et puis là il m'a annoncé ...

Q. Vous vous êtes rendu vers quelle heure au bureau à peu près ? R. Vers les dix (10) heures, entre les dix (10) heures et dix heures et demie durant la haute-messe il m'a parlé de question de grève, qu'il devait aller en grève.

Q. Comment vous a-t-il dit cela ? Expliquez... R. Il dit «nous sommes dans le moment, monsieur Roger Bédard est en communication avec Pittsburgh pour avoir une approbation pour aller en grève. Alors, on a discuté de la grève un peu, demandé pourquoi il y allait si tôt parce qu'il avait toujours dit qu'il irait seulement après Noranda. Il a dit «ben, c'est parce que moi, j'ai été congédié, et puis que toi, tu vas l'être demain et cent vingt-cinq (125) autres vont suivre ». De part et d'autres, nous avons discuté différentes affaires de la grève et puis je lui ai dit "c'est ben de valeur, je suis obligé de m'absenter, il faut que j'aille à Gaspé conduire ma femme à l'hôpital » et je suis parti.

Q. Vous êtes parti pour Gaspé, lorsque vous êtes allé à la messe le matin, est-ce qu'on y a annoncé une assemblée pour le soir? Vous souvenez-vous... R. Non, pas de mémoire.

Q. À quel moment avez-vous entendu parler qu'il y était pour avoir une assemblée le dimanche soir ? R. C'est le moment où Théo me l'a annoncé dans l'avant-midi.

Q. Est-ce que Théo Gagné vous a dit qu'il était au courant qu'il y aurait une grève de déclarée ce soir-là ? $\mathbf{R}$. Oui, il m'a dit «nous devons déclarer la grève ce soir, mais nous attendons l'approbation de Pittsburgh toujours ».

Q. Mais est-ce qu'il vous a dit qui était au courant de ça à Murdochville ? R. Il a dit que personne était au courant, et puis là, il m'a dit de ne pas en parler à personne parce qu'il ne voulait pas que ça se sache avant l'assemblée du soir.

Q. Alors, vous êtes parti pour Gaspé conduire votre femme à l'hôpital ? R. Oui. 
Notons que l'appelante a son bureau-chef à Pittsburgh, aux États-Unis, d'où elle dirige et contrôle financièrement les locaux qui lui sont affiliés au Canada, comme aux États-Unis.

Avant même la réunion du comité des officiers, une équipe régulière d'une vingtaine d'ouvriers préposée au concassage fait défaut de se présenter à l'usine à trois heures p.m. Tard dans l'après-midi, les membres du comité se réunissent et adoptent une résolution recommandant l'entrée en grève pour le soir à minuit. Eugène Lapointe, l'un des stewards, assiste à cette assemblée du comité exécutif. $\mathrm{Au}$ procès, il est appelé par l'appelante comme témoin. En contre-interrogatoire, on lui demande :

Mais pourquoi à ce moment-là le comité aurait-il décidé la grève quand M. Gagné n'aurait pas encore vu Brissenden et qu'il devait le voir le lundi matin et que d'après vous-même, vous aviez confiance que $M$. Brissenden arrangerait ça ?

À cette question, il répond :

Bien, parce qu'il y a eu une rumeur, ils ont arrivé avec une rumeur qu'il y aurait 100 ou 125 hommes de congédiés quand même, c'est là que le comité a décidé d'appeler une assemblée générale pour entrer en grève.

Bédard arrive en retard à cette assemblée du comité de l'Exécutif. On l'avise de l'adoption de la résolution qu'il accepte de communiquer lui-même à l'assemblée générale des membres le dimanche soir. Gagné préside cette assemblée générale. Lui et Bédard déclarent que vu le congédiement du président du syndicat, Théo Gagné, et les menaces de congédiements qui affectent de nombreux officiers, stewards et membres du Local, la grève est devenue inévitable. Sur ces déclarations de Bédard et Gagné, voici d'ailleurs quelques témoignages :

\section{Abraham Arsenault :}

Q. Ėtes-vous revenu le même dimanche à Murdochville? R. Oui, je suis revenu vers l'heure du souper, soit six à sept heures.

Q. Et êtes-vous allé à l'assemblée ? R. Oui.

Q. Est-ce que vous êtes arrivé au commencement ou avant l'ouverture ? R. Avant que les orateurs parlent.

Q. Je vois. Vous souvenez-vous qui a été le premier orateur ce soir-là ? R. Oui, Théo Gagné.

Q. Je vois. Vous souvenez-vous ce qu'il a dit ? R. Il a commencé par annoncer aux gars que l'assemblée avait été convoquée vitement: pour la raison qu'on devait partir en grève le même soir et que les raisons étaient qu'il avait été congédié et que moi-même j'y serais congédié et cent vingt-cinq (125) autres qui suivraient dans le courant de la semaine.

Q. Et quand ils vous ont mentionné là, qu'est-ce qu'il a fait ? Qu'est-ce qu'il vous a demandé de faire quand il a dit que vous, vous seriez: congédié ? R. Il m'a demandé de me lever pour démontrer aux. gens, aux employés que j'étais un de ceux qui allaient être congé.. diés «vous connaissez tous Abraham Arsenault»... 
Q. À quel moment Bédard est-il arrivé à l'assemblée ? R. Il est arrivé un peu après, pas très très longtemps.

Q. Et alors, est-ce que Gagné a dit d'autres choses au sujet de Bédard avant l'arrivée de Bédard ? R. Il nous a dit que monsieur Bédard était en communication avec Pittsburgh, qu'il attendait l'approbation de Pittsburgh pour aller en grève et puis la question des secours de grève qui devaient être financés par le bureau-chef de l'Union des Métallos Unis d'Amérique.

Q. À un moment donné, vous dites que Bédard est arrivé, qu'est-ce qu'il a fait? R. Aussitôt qu'il est arrivé, là, peu de temps après, monsieur Gagné lui a donné la parole et il nous a affirmé qu'il avait eu l'approbation du bureau-chef de déclarer la grève qu'il fallait que la grève soit déclarée immédiatement et puis que on pouvait être assuré de «toute 》 le support des Métallos Unis d'Amérique.

Q. Est-ce qu'il a parlé des fournaises de la compagnie ? R. Là, dans son discours, il nous a dit que c'était le moment, que le moment était venu d'aller en grève immédiatement avant le quart qui rentrait à onze heures pour empêcher le quart de onze heures de rentrer, par rapport que les fournaises étaient «pleins》 de cuivre et que si la compagnie cédait pas, tout gèlerait là et qu'il s'en sacrait pas mal.

Q. Est-ce qu'il a été question dans le discours de Bédard des obligations que les employés avaient à rencontrer les dettes qu'ils pouvaient avoir à payer et qu'est-ce que Bédard a dit à ce sujet ? R. Y'a eu plusieurs questions de posées sur le sujet. Ceux qui avaient des automobiles sur les finances, d'autres, des meubles sur les finances, d'autres qui occupaient les maisons de la compagnie qui devaient payer loyer, d'autres qui avaient acheté des maisons, tout ce qui comprenait la finance. Y'a eu des questions de posées là-dessus, chacun avait son problème, il leur a dit de ne pas s'inquiéter, ceux qui étaient en grève, que les finances pouvaient pas venir rien leur enlever. À Noranda, ils avaient été en grève pendant six ou sept mois et que personne n'avait perdu rien et qu'il les assurait qu'ils ne perdraient rien.

Q. Est-ce que des questions lui ont été posées au sujet du montant que les grévistes recevraient par semaine ? R. Oui, plusieurs questions lui ont été posées à ce sujet-là, il lui a dit que tout dépendait du nombre de dépendants.

Q. Est-ce qu'il a toutefois mentionné certains chiffres ? R. Le seul chiffre qu'il a mentionné, à ma connaissance, c'est qu'il avait l'appui du bureau-chef pour environ un million et demi pour les deux, Noranda et Gaspé Copper.

Q. Alors, qu'est-ce qui s'est passé après ces explications données par Bédard à l'assemblée? R. Après, y'a eu quelqu'un qui ont demandé si le vote de grève était encore bon, vu qu'il avait été pris au mois de septembre; d'autres voulaient qu'il y ait un autre vote secret de pris et puis il a dit que ça serait inutile qu'il fallait aller en grève le même soir.

Q. Et là, qu'avez-vous fait, là ? Qu'est-ce que l'auditoire a fait après ça ? R. Là, ça s'est levé, ça discutait un peu entre eux-autres, je me suis levé, j'ai demandé à monsieur Gagné s'il ne serait pas bon 
d'avertir la compagnie avant d'aller en grève parce que d'après le petit peu d'intelligence que j'avais, j'avais entendu dire qu'il fallait avertir une compagnie avant d'aller en grève. Il a dit que que «c'était inutile, qu'il fallait y aller immédiatement», c'était toujours les mêmes réponses qu'il me donnait.

Q. Vers quelle heure cette assemblée-là s'est-elle terminée ? R. Alentour de dix heures, dix heures et quart, parce qu'il nous disait qu'il fallait se rendre à la barrière vers les dix heures et vingt, dix heures et demie pour pas que le «shift» de onze heures rentre.

Normand Boudreau, électricien et membre de l'Union :

Q. J'en arrive à l'assemblée du dimanche soir, le dix (10) mars; vous y avez assisté, n'est-ce pas? R. Oui.

Q. Voulez-vous dire à la Cour ce qui s'est passé et avec le plus de détails possibles, ce que vous y avez entendu par les orateurs ? R. Bien, j'ai eu, j'ai entendu dire qu'il y avait l'assemblée, j'étais à la messe le dimanche matin, je me suis rendu là, y'avait Bédard. Bédard a arrivé en retard un peu.

Q. Vous voulez dire à l'assemblée ou à la messe ... R. À l'assemblée.

Q. Et puis ... R. C'est Théo Gagné qui présidait l'assemblée, et puis y'a eu des discussions à l'effet de Théo Gagné qui avait été «clairé ». Il était supposé y avoir une liste de cent vingt-cinq (125) tous des gars de l'Union, les principaux «stewards».

Q. Une liste de cent vingt-cinq (125) ? R... qui étaient pour être congédiés.

Q. Qui a dit ça ? R. Ca, c'est Bédard, Bédard a dit ça plusieurs fois.

Q. À l'assemblée ? R. Oui, Et puis là, il a dit, je me rappelle qu'il a dit que la seule arme, c'était d'aller en grève. On avait pas d'autres choix. Et puis là y'en a dans la salle qui n'ont pas aimé ça, ils ont levé, ils ont dit que c'était entendu qu'on était pour partir en grève vingt-quatre (24) heures après Noranda. Y'en a un qui s'est levé et qui a mentionné ça à Bédard. Bédard a viré ça en ridicule, il a dit «vingt-quatre (24) heures avant ou après, ça ne fait pas de diffé. rence, il a dit, à dix heures, ce soir-là, la grève. Commencez d'aller aux barrières de la mine et ne pas laisser entrer personne ni «staff 》 ni travaillant, ni personne.

Q. A-t-il été question d'autres choses dans le discours de Bédard ce soir-là ? R....

Q. ... au sujet de la grève ? R. Y'a eu beaucoup de choses au sujet de la grève, mais là, je me rappelle pas toutes.

Q. A-t-il été question d'allocations que pourraient recevoir les grévistes s'il y avait grève ? R. Oui, on était supposé d'avoir un mil. lion, je pense de Pittsburgh, cinq cent mille piastres $(\$ 500,000)$ de Toronto. C'était pour partir, pour déclencher la grève et puis chaque famille aurait une allocation. Plus grosse la famille, plus grosse l'allocation. 
Sylvio Pascualeto, mécanicien et membre de l'Union :

Q. Voulez-vous dire à la Cour ce dont vous vous souvenez de cette assemblée-là ? R. Bien, ce que je me souviens, d'après l'assemblée, je sais que monsieur Bédard n'était pas là tout de suite au début de l'assemblée, Théo Gagné, je pense a dit quelques paroles, mais je ne me rappelle pas du tout les paroles de Théo. Là-dessus, toujours monsieur Bédard est arrivé, il s'est excusé du retard, il s'est excusé du retard qu'il avait apporté, mais la faute, je pense a passé sur un nommé Raymond Lapointe. Il attendait monsieur Lapointe, la température n'était pas trop clémente, et vu qu'il descendait en voiture, ça fait que monsieur Bédard nous a dit : «Je m'excuse pour ça...pardon», c'est lui-même qui a ouvert l'assemblée, monsieur Bédard a ouvert l'assemblée, et puis là ...

Q. ... qu'est-ce qu'il vous a dit ? R. Sa question primordiale a été, il nous a dit "messieurs, vous savez comme moi que vendredi donc le huit (8), je pense - monsieur Théo Gagné a été licencié de Gaspé Copper Mines. Mais ce n'est pas Théo Gagné le plombier qui a été licencié, c'est Théo Gagné, le président de votre local ». «Donc, comme vous le voyez, Gaspé Copper Mines est une compagnie sans coeur...» encore qu'il nous a dit, et puis «je pense que le moment d'agir est arrivé, messieurs...» Comment est-ce qu'il nous a dit ça ... «l'heure a sonné, l'heure est critique, donc il n'y a plus qu'une seule chose à faire, c'est la grève ». Là-dessus, il y a eu plusieurs questions qui se sont soulevées à propos de l'aide. Ca fait que monsieur Bédard nous a dit qu'il avait été en relation avec la tête, c'est-à-dire Pittsburgh ... je ne sais pas, quelque chose comme ça, toujours, et puis que nous aurions le support moral et financier de la C.I.O.

Q. Ensuite...? R. Là-dessus, y'a eu plusieurs questions de soulevées, si je me rappelle bien...

Q. Est-ce qu'il y a eu des questions au sujet des dettes que les employés devaient à ce moment-là et qui deviendraient dues durant la grève, s'il y avait grève ? $R$. Oui, il nous a dit... la première question primordiale «de ne s'inquiéter d'aucune dette, que l'International elle-même paierait les intérêts pour ceux qui avaient des meubles à payer, des voitures .... que tout était arrêté, de n'avoir aucune crainte, que personne ne serait saisi, et que lui prendrait les arrangements nécessaires pour l'intérêt, c'est-à-dire, payer l'intérêt à toutes ces compagnies, de ne s'inquiéter d'aucune façon. Deuxièmement, que nous aurions les dûs de l'Union, qu'on attendait plusieurs messieurs, justement de la C.I.O. pour nous remettre les dûs de l'Union.

À la fin de l'assemblée, Bédard organise le piquetage; on forme des groupes, on en nomme les chefs et on se rend aux barrières de l'usine pour en bloquer tous les accès.

En Cour supérieure et en Cour d'appel, on a noté qu'au cours de cette assemblée générale, convoquée précipitamment pour obtenir d'une minorité présente des travailleurs, une décision hâtive favorisant le déclenchement immédiat de la grève pour le soir même, Bédard et Gagné avaient pris soin de ne pas les informer du caractère temporaire, non définitif et de la véritable raison de la mise à pied de 
Gagné et de ne pas les informer du fait que Gagné lui-même, précisément en vue de discuter d'un autre poste ou emploi à l'usine, avait déjà convenu d'un rendezvous avec le directeur général pour le lundi. On a aussi noté que, de plus, on a alors dénaturé la raison, le caractère et la date de la réduction progressive de personnel que la Direction avait, en février, envisagée comme possible à la fin des travaux faits sous terre et après l'installation de la machinerie lourde et qu'on a représenté plutôt que la Direction avait décidé d'un congédiement massif et immédiat d'officiers, stewards et membres de l'Union. Ainsi donc par le procédé du silence, joint à celui de la fausse représentation, on aura voulu subtilement former, soulever l'opinion et assurer que les travailleurs présents à cette assemblée générale soient induits à conclure que par le renvoi définitif du président du syndicat et autres renvois imminents visant les membres les plus actifs du Local, la compagnie intimée entendait détruire le syndicat de l'appelante et que dès lors et dans une réaction de révolte, l'assemblée approuverait le déclenchement immédiat de la grève.

Tels sont en somme les faits et circonstances essentiels retenus de la preuve, tant par le juge au procès, M. le juge Lacoursière, - qui, pour ainsi dire, a vécu avec cette cause pendant plus d'un an, a vu les témoins, entendu toute la preuve, tous les témoignages, les a pesés, évalués, en a considéré les conflits, le tout au regard du degré de crédibilité qu'il a jugé et, en fait, déclaré accorder ou refuser aux principaux témoins des parties, - que par les juges de la majorité en Cour d'appel.

Au stade du présent appel, l'appelante ne conteste plus que, une fois la grève déclenchée, elle l'ait dirigée et financée et que ses représentants aient été directement ou indirectement impliqués dans certains des actes illégaux qui s'ensuivirent. Elle représente cependant, - et c'est là, à mon avis, une prétention, dont le bien fondé est essentiel au succès de l'appel, - que c'est sans son intervention ou celle de ses représentants, mais dans un mouvement spontané de révolte provoqué par la mise à pied de Gagné et la menace de congédiements massifs et imminents des membres les plus actifs du Local que les travailleurs présents à l'assemblêe générale approuvèrent la décision de l'entrée en grève ce soir-là. C'est là une question de fait. Et la prétention de l'appelante sur ce point a été rejetée comme non fondée, tant par le jugement de la Cour supérieure que par celui de la Cour d'appel. Pour sa part, le juge au procès s'exprime ainsi sur la question :

Le silence de Bédard et de Gagné devant l'assemblée, sur cette entrevue proposée par M. Brissenden, pour le lundi matin, ne peut s'expliquer autrement que par l'existence d'un plan de grève déjà arrêté par les dirigeants et qu'il fallait faire approuver, avant que les employés réalisent que cette histoire de congédiement était pour le moins exagérée. Pourquoi cette précipitation à convoquer, pour le soir même, une assemblée qui ne pouvait réunir qu'une faible proportion des travailleurs, puisque un grand nombre d'eux se trouvaient en dehors de Murdochville, où ils ne devaient revenir que le lendemain matin pour leur ouvrage?

Comme le congédiement de Gagné seul aurait bien pu ne pas être jugé un motif suffisant par les travailleurs pour aller en grève, on insista surtout sur le renvoi probable de 125 employés, dont les «stewards » de l'union. Il a été démontré que cette dernière rumeur n'était que pure invention. 
Il serait bien naïf de croire que la décision de grève fut le résultat d'un mouvement spontané de révolte de la part des employés contre la compagnie, et que la défenderesse (United Steel-Workers of America) a dû, bien involontairement, en subir les conséquences. La preuve établit nettement le contraire.

En Cour d'appel, M. le juge Taschereau cite, avec approbation, ces différents extraits du jugement de $M$. le juge Lacoursière. Il conclut :

Donc, à mon avis, une seule conclusion s'impose, c'est que, contrairement à ce que prétend l'appelante, cette grève n'a pas été la réaction spontanée au congédiement de Théo Gagné, le président du Syndicat, mais bien le résultat d'un plan ourdi depuis quelque temps déjà.

M. le juge Hyde déclare ce qui suit: (Traduction)

Après tout, Gagné était le principal intéressé. S’il était disposé à laisser un malentendu généralisé se répandre parmi ses compagnons de travail, ce n'était pas la faute de la compagnie. Il savait très bien qu'il était convoqué chez le directeur le lundi après-midi et il connaissait le but de cette entrevue. La prétendue raison de la grève, telle que donnée à la réunion du Local, le dimanche, était de protester contre son congédiement. Cependant il ne fallut que peu de temps pour que le but avoué de la poursuite de la grève devienne la reconnaissance du syndicat et la conclusion d'une convention (voir, à titre d'exemple, le Bulletin «Grève 》 du Local no 4881, 27 mars 1957, pièce C-189).

L'autre cri de ralliement à la réunion du dimanche était la fausse rumeur relative au renvoi imminent de 125 autres employés. Il n'y a eu aucune tentative, ni de la part de Bédard, ni de celle de Gagné de contrôler la véracité de cette rumeur auprès de la direction.

De plus, même si la compagnie avait enfreint la Loi des relations ouvrières en congédiant Gagné à cause de son activité syndicale, ce renvoi n'aurait pas été une cause déterminante des dommages résultant de la grève. Le congédiement illégal, à supposer qu'il le soit, était indépendant et distinct de la déclaration illégale de grève et des autres actes illégaux qui ont suivi. Il est important de faire remarquer ici que Gagné n'a pas accusé la compagnie d'une violation de la Loi, ni intenté d'action en dommages pour congédiement injustifié, alors que ces recours lui étaient manifestement permis si la position de l'appelante était soutenable.

La Cour d'appel et la Cour de première instance ont toutes deux conclu que la grève n'a pas résulté d'un mouvement spontané de révolte; elles ont jugé que l'appelante a saisi l'occasion de cette mise à pied pour en faire un prétexte de grève et ainsi voiler et mettre à exécution un plan depuis longtemps arrêté pour contraindre la compagnie intimée à reconnaître le Local de Murdochville comme représentant accrédité aux fins de la négociation du nouveau contrat de travail. En somme, on a jugé que l'appelante avait la responsabilité exclusive de la grève.

En présence de l'accord des deux Cours sur cette conclusion sur les faits et au regard du principe régissant la fonction d'une cour d'appel en pareil cas, principe bien connu auquel le savant procureur de l'appelante a d'ailleurs référé au tout début de l'audition, pour indiquer qu'il était conscient de l'obstacle qui 
devait être écarté, pour le succès de l'appel, - nous ne devons intervenir que s'il apparaît que cette conclusion est, suivant l'expression consacrée par la jurisprudence, fondamentalement erronée. À mon avis, il n'a pas été démontré que tel est le cas.

La règle de non-intervention reçoit ici son application.

Cette conclusion des deux Cours, quant à ce qui constitue en fait la véritable cause de la décision de faire la grève le 10 mars, dispose, à mon avis, des autres moyens soumis par l'appelante ou retenus par $M$. le juge Brossard pour imputer à la compagnie intimée une part de responsabilité. Il convient, cependant, d'y référer brièvement.

Dans une dissidence, remarquable par le soin avec lequel il traite du problème des relations ouvrières et de ses aspects juridiques, le savant juge conclut, à l'instar de ses collègues, que, par les actes de ses représentants, l'appelante «a provoqué, aidé et conseillé la décision de faire la grève ».

D'autre part, et en ce qui concerne la mise à pied de Gagné, il émet l'opinion que cette mise à pied fut faite en violation des dispositions de la Loi sur les Relations Ouvrières stipulant que tant que ne sont pas réalisées les conditions requises pour que prenne fin l'interdiction de la grève, les conditions de travail des salariés ne doivent pas être changées sans leur consentement et que, «dans les circonstances, la mise à pied de Gagné constituait une violation du contrat de travail entre lui et la compagnie». En ce qui a trait aux procédures de prohibition instituées par l'intimée, le sursis par elle obtenu pour empêcher la Commission des Relations Ouvrières de poursuivre les procédures sur la demande d'accréditation vu son refus de fournir les informations demandées, et en ce qui concerne la suspension de l'adjudication sur l'inscription en droit totale de la Commission suivant l'accord intervenu entre elle et l'intimée, M. le juge Brossard est d'avis que ces procédures constituent un abus du recours de droit parce que, dit-il, nonobstant leur caractère régulier, elles n'avaient d'autre but que celui de faire obstacle à la reconnaissance syndicale du Local de l'appelante. Enfin, référant au refus donné au syndicat par l'intimée, le 1er octobre 1956, d'entreprendre des négociations, vu que les procédures relatives à l'accréditation étaient pendantes en Cour supérieure, il déclare que ce refus constituait une faute et une provocation au Local de mettre à exécution le vote de grève qu'il avait pris le 20 septembre. Sur le tout, le savant juge conclut qui si ces procédures judiciaires et ce refus de négocier n'ont pas entraîné immédiatement une grève que le Local avait décidé en principe le 20 septembre 1956, «ils ont néanmoins été la cause que la mise à pied de Gagné le 8 mars 1957 a pris une importance et eut pour conséquence la déclaration de grève le 10 mars 1957. 》

Avec le plus grand respect, je ne puis souscrire à ces vues.

Quant à la mise à pied de Gagné, je dirais qu'il ressort de l'extrait ci-dessus de son propre témoignage, que rien n'était définitif et que la continuation de ses services à la compagnie était l'objet de l'entrevue dont il avait convenu avec le directeur général pour lundi. De plus, bien que d'avis que Gagné fut remercié de ses services, M. le juge Brossard n'hésite pas à affirmer que «... il n'y a aucune preuve qui puisse permettre de conclure que Gagné fut remercié de ses services. 
à raison du fait qu'il était membre et président du Local $4881 \ldots$... Prenant pour acquis que cette mise à pied était définitive et constituait une violation du contrat de travail entre Gagné et l'intimée, - ce que les deux Cours ont jugé ne pas être le cas, - je dirais, comme M. le juge Hyde, qu'il s'agirait là d'un acte séparé, distinct de la déclaration illégale de la grève, susceptible de donner ouverture à un recours sous le droit commun ou sous la Loi sur les Relations Ouvrières, mais n'ayant pas, d'autre part, de relation avec le dommage causé par la grève.

Je ne puis davantage retenir que l'intimée ait commis un abus de recours de droit causant la décision de faire la grève le $\mathbf{1 0}$ mars. Tous les arrêts de la Cour de cassation, déclare Savatier dans son Traité de la responsabilité civile en droit français, Tome 1, p. 83 , nos 65 et seq., s'accordent à reconnaître que l'action en justice, c'est-à-dire le droit de soumettre au juge ses prétentions est un droit dont l'exercice n'entraîne, en principe, aucune responsabilité, même si ces prétentions sont éventuellement rejetées. Sans doute, l'exercice de ce droit peut dégénérer en faute susceptible, si dommageable, d'entraîner une condamnation en dommages intérêts dans le cas où cet exercice constitue un acte de mauvaise foi ou s'il est tout au moins le résultat d'une erreur grossière équipollente à dol. Encore faut-il que la mauvaise foi soit prouvée ainsi que l'exigent les dispositions de l'article 2202 C.C. :

2202. La bonne foi se présume toujours et c'est à celui qui invoque la mauvaise foi de la prouver.

Que les procédures en question et principalement la suspension de l'adjudication sur l'inscription en droit totale de la Commission aient eu pour effet de faire obstacle à la reconnaissance syndicale du Local de l'appelante, il ne s'ensuit pas qu'il faille conclure à la mauvaise foi de la Commission et de l'intimée qui convinrent de cette suspension et à la mauvaise foi du tribunal qui donna suite à leur accord d'attendre le jugement de la Cour d'appel dans deux causes où une même question se présentait. Rien au dossier, a-t-on noté en Cour d'appel, n'indique qu'on ait fait de la part du syndicat de l'appelante des démarches judiciaires pour se plaindre de cette suspension, demander l'adjudication de l'inscription en droit ou contester l'émission du bref de prohibition. Une conclusion de mauvaise foi conduit inévitablement, il me semble, à mettre en question le bien fondé de la déclaration de M. le juge Morin à l'effet «qu'il n'y a eu dans toute cette affaire aucun retard intentionnel non seulement de la part du tribunal mais aussi de la part des procureurs des parties dans la présente cause. »Ces considérations sur les procédures valent également, à mon avis, quant au refus que l'intimée donnait le ler octobre 1956 en raison du fait que les procédures relatives à l'accréditation étaient pendantes en Cour supérieure.

Aussi bien, étant d'opinion qu'il n'a pas été démontré que le jugement de la Cour supérieure et le jugement de la Cour d'appel soient fondamentalement erronés en ce qui concerne les faits, je ne saurais conclure que l'intimée doit supporter une part du préjudice lui résultant du fait de la grève.

Ces conclusions sur les faits justifient, en droit, le jugement de la Cour d'appel qui a confirmé celui de la Cour supérieure condamnant l'appelante à la réparation de l'entier préjudice subi par l'intimée, tant par suite du fait de la grève que par suite des autres actes délictuels ou criminels qui s'ensuivirent. L'objet fondamental 
de la Loi sur les Relations Ouvrières, - comme d'ailleurs celui du Code du Travail qui fut substitué à cette loi, - est d'assurer la paix industrielle. Cette loi ne modifie par les principes généraux qui, sous le droit commun, régissent la responsabilité civile en matière délictuelle et qui imposent à celui ou ceux qui, comme en l'espèce, causent des dommages à autrui ou par les actes délictuels ou criminels de ceux dont ils ont le contrôle, l'obligation de réparer le préjudice en résultant. Notre droit n'admet pas et ne saurait admettre, sans lui-même se détruire et faire place à l'anarchie, le recours à l'intimidation, l'obstruction, actes de sabotage, actes de dynamitage, de violence, en somme le recours à la force, comme moyens légitimes d'obtenir la reconnaissance du droit auquel le justiciable peut prétendre si manifeste que soit le bien fondé de sa prétention.

Pour ces motifs, je rejetterais l'appel avec dépens.

\section{LE JUGE HALL :}

Je suis d'accord avec mon collègue le Juge Fauteux que les conclusions du savant Juge de première instance, confirmées par les Juges de la Cour du Banc de la Reine, quant à la responsabilité de l'appelante sont correctes, et que la preuve les justifie pleinement.

Toutefois, je suis d'avis que l'intimée est elle aussi, en partie, responsable de la grève qui s'est produite dans les circonstances relatées dans la preuve. Je suis d'accord avec le point de vue adopté par le Juge Brossard, de la Cour du Banc de la Reine, selon lequel la mise à pied de Gagné a été l'une des causes de la grève et, sous ce rapport, je souscris à ses motifs savants et complets, donnés à la Cour d'appel et reproduits à (1967) B.R. 487, aux pages 565 à 568.

À la Cour du Banc de la Reine, voici ce que dit le Juge Hyde, dans la partie de ses motifs intitulée (traduction) Le congédiement de Gagné :

« Il est clair, et cela devait être évident pour la Compagnie que les membres du syndicat de Gagné s'offusqueraient d'une telle maladresse dans sa mise à pied, vu spécialement la situation de tension qui existait entre elle et le local no 4881. La Compagnie devait savoir qu'il y avait déjà eu des grèves illégales et Brissenden aurait dû prendre toutes les précautions nécessaires pour éviter toute possibilité de malentendu à l'occasion d'un acte aussi risqué que le congédiement ou la mise à pied du président du syndicat »;

dans une autre partie, il ajoute sous le titre (traduction) Le plaidoyer de justification et provocation:

«Bien que j'aie dit en examinant la mise à pied de Gagné que je trouvais qu'elle avait été maladroite jusqu'à la stupidité, en tenant compte particulièrement de la situation de tension qui existait entre la Compagnie et le local no 4881, je ne puis, du fait que je conclus que Gagné savait très bien à quoi s'en tenir sur sa situation, caractériser cette maladresse comme une faute, si faute il y a. Après tout, Gagné était le principal intéressé. S'il était disposé à laisser un malentendu généralisé se répandre parmi ses compagnons de travail, ce n'était pas la faute de la compagnie. Il savait très bien qu'il était convoqué chez le directeur le lundi après-midi et il connaissait le but de cette 
entrevue. La prétendue raison de la grève, telle que donnée à la réunion du Local, le dimanche, était de protester contre son congédiement. Cependant il ne fallut que peu de temps pour que le but avoué de la poursuite de la grève devienne la reconnaissance du syndicat et la conclusion d'une convention (voir, à titre d'exemple, le Bulletin 《Grève » du local no 4881, 27 mars 1957, pièce C-189) 》;

il dit enfin, dans la partie intitulée (traduction)

\section{CONCLUSIONS}

«Toute cette affaire est malheureuse et tragique. Bien que je ne puisse approuver la conduite de l'intimée dans ses relations avec sa main-d'oeuvre, je suis, en droit, obligé de rejeter tout le tort sur l'appelante. Aucune faute de la part de l'intimée ne justifiait les actes illégaux auxquels l'appelante a pris part. De toute façon, il n'y avait aucune relation de cause à effet entre cette faute, si faute il y a, et les dommages causés à l'intimée. »

Je suis tout à fait d'accord avec la conclusion que la faute de l'intimée ne justifiait pas les actes illégaux auxquels l'appelante a pris part. Il ne pouvait y avoir aucune justification légale des actes illégaux de l'appelante. Le Juge Brossard ne soutient pas cela. À mon avis, le congédiement illégal de Gagné a été de lui-même, indépendamment des agissements illégaux de l'appelante, une cause contributive du déclenchement de la grève. Il n'y a pas de doute que le local no 4881 se préparait à la grève et que l'intimée le savait parfaitement. Elle avait déjà emmagasiné des provisions et aménagé des logements temporaires pour les non-grévistes qui demeureraient sur les lieux, en cas de grève. Il est fort probable qu'il y aurait eu grève tôt ou tard, mais pas nécessairement cette fin de semaine-là. Peut-être même il n'y aurait pas eu de grève du tout après qu'on aurait connu le règlement intervenu à Noranda, si l'intimée n'avait pas elle-même mis le feu aux poudres en congédiant illégalement Gagné et cela malgré l'intention que pouvait avoir Brissenden de reprendre Gagné le lundi. Le mal était fait quand on a remis à Gagné son avis de congédiement.

Je ne puis admettre qu'il n'y a pas de relation de cause à effet entre le congédiement de Gagné et une partie des dommages subis par l'intimée. Elle a subi ces dommages sous deux chefs : (1) par l'effet de la grève elle-même, soit par une diminution de la production et autres pertes dues à l'arrêt de travail lui-même; (2) par les pertes résultant des actes illégaux et du sabotage qui se sont produits, selon les conclusions du savant Juge de première instance. La relation de cause à effet entre les dommages subis sous le premier chef et le congédiement illégal de Gagné est évidente parce que ce congédiement a été l'une des causes de la grève, mais il n'y a pas de telle relation pour les dommages subis sous le second chef. En toute déférence, le Juge Hyde me semble avoir oublié cette distinction dans l'énoncé de sa conclusion finale, que j'ai cité.

En conséquence, j'accueillerais l'appel en partie en tenant l'intimée responsable à 25 p. 100 et en réduisant dans cette proportion les dommages sur lesquels il y a eu accord. L'appelante a droit à ses dépens en cette Cour. 